\title{
PSX: Protein-Solvent Exchange - Software for calculation of deuterium-exchange effects in SANS measurements from protein coordinates
}

\author{
Martin Cramer Pedersen, ${ }^{a}{ }^{*}$ Yong Wang, ${ }^{b}$ Frederik Grønbæk Tidemand, ${ }^{a}$ \\ Anne Martel, ${ }^{c}$ Kresten Lindorff-Larsen $^{b}$ And Lise Arleth ${ }^{a}$ \\ ${ }^{a}$ Niels Bohr Institute, University of Copenhagen, Denmark, ${ }^{b}$ Linderstrøm-Lang \\ Centre for Protein Science, Department of Biology, University of Copenhagen, \\ Denmark, and ${ }^{c}$ Large Scale Structures Group, Institut Laue-Langevin, France. \\ E-mail: mcpe@nbi.ku.dk
}

hydrogen/deuterium exchange; small-angle neutron scattering; biophysical samples; solvent exposure; bioinformatics

\begin{abstract}
Recent developments in neutron scattering instrumentation and sample handling have enabled studies of more complex biological samples and measurements at shorter exposure times. To extract most information from such experiments it is thus desirable to determine accurate estimates of deuteration levels. We introduce and document software for exploring the effect of hydrogen-deuterium exchange for proteins solubilized in $\mathrm{D}_{2} \mathrm{O}$ as well as the underlying bioinformatical models. The software aims to be generally applicable for any atomistic structure of a protein and its surrounding environment, and thus captures effects of both heterogenous exchange rates throughout
\end{abstract}


the protein structure and by varying the experimental conditions such as $p H$ and temperature. The paper concludes with examples of applications and estimates of the effect in typical scenarios emerging in small-angle neutron scattering on biological macromolecules in solution. We find that the common assumption of $90 \%$ exchange is in many cases a strong overestimate with the rapid sample handling systems currently available. Source code for the presented software is available from an online repository in which it is published under version 3 of the GNU publishing license.

\section{Introduction}

Small-angle neutron scattering (SANS) experiments are emerging as a powerful source of information about the structure and dynamical properties of macromolecules such as proteins. In order to extract as much information as possible from such experiments during, for example, model refinement, it is often necessary to provide external information such as scattering lengths, molecular volumes, and similar physical parameters. SANS experiments are often carried out using either $\mathrm{D}_{2} \mathrm{O}$ or $\mathrm{H}_{2} \mathrm{O} / \mathrm{D}_{2} \mathrm{O}$ mixtures providing opportunities for varying contrast between different parts of the sample, due to the stark difference in scattering properties between protons and deuterons. When a protonated protein is exposed to $\mathrm{D}_{2} \mathrm{O}$, a number of protons in the protein will exchange with the deuterons in the solvent over a timescale that may be comparable to that of the experiment. Thus, for samples and experiments such as these, this exchange of protons must be accounted for in order to refine accurate models of the samples in question .

These considerations become increasingly relevant for proteins with domains shielded from the solvent such as e.g. a membrane protein in a phospholipid bilayer nanodisc (Kynde et al., 2014; Johansen et al., 2018) or detergent micelle (Midtgaard et al., 2018). The recent developments of so-called "invisible" carrier systems that 
match the scattering properties of the associated solvent, have been used for solubilizing membrane proteins for solution SANS experiments; in particular the socalled match-out deuterated detergent micelles (Midtgaard et al., 2018) or stealth nanodiscs (Maric et al., 2014; Josts et al., 2018), add further relevance to the considerations.

On a similar note, in-line size exclusion chromatography (SEC) for BioSANS has recently emerged as a promising technique for investigating particularly complicated and fragile biological samples of interest (Jordan et al., 2016; Johansen et al., 2018). When employing this technique, samples are exposed to the solvent, in many cases $\mathrm{D}_{2} \mathrm{O}$, only minutes before being irradiated by the neutron beam, meaning that the exchange process is far from equilibrium.

In this paper, we present an approach to and software for more accurately estimating the hydrogen-to-deuterium exchange (also commonly known as protium-deuterium exchange) in a protein structure as a function of the time in which the protein has been exposed to $\mathrm{D}_{2} \mathrm{O}$ using a well established model from the world of structural bioinformatics. The software focuses on the exchange of the backbone hydrogens, as these are the most relevant exchanges in the context of neutron scattering experiments (Figure 1). Specifically, we divide hydrogens into three groups: (1) non-exchanging, carbon-bound hydrogens, (2) slowly-exchanging backbone hydrogens that exchange with the solvent at timescales relevant for neutron scattering experiments on biological samples, and (3) the remaining rapidly-exchanging side chain hydrogens.

Some existing software packages such as CRYSON (Svergun et al., 1998) and ISIS' Biomolecular Scattering Length Density Calculator (ISIS, 2019) assume a homogeneous deuteration level throughout the molecule (defaulting to $90 \%$ of the backbone hydrogens and $100 \%$ of the rapidly-exchanging hydrogens in CRYSON and $90 \%$ of the rapidly exchanging hydrogens plus the backbone hydrogens in ISIS' Biomolecular 
Scattering Length Density Calculator); thus not accounting for actual solvent exposure and the geometry of the protein. Other software packages such as e.g. SASView (The SASView Project, 2019) or WillItFit (Pedersen et al., 2013) implicitly rely on accurate estimates of scattering lengths.

\section{Empirical model for hydrogen-deuterium exchange}

We here describe an approach to estimate the level of deuteration of each of the exchangeable backbone amide protons using the structure of the protein. We do so via estimating both the so-called intrinsic exchange rate constants from the amino acid sequence, and the perturbation away from these intrinsic rates in a give conformation of the protein. We assume that hydrogen-deuterium exchange can be described by the well-established Linderstrøm-Lang model, in which the exchange of a buried or otherwise protected proton with a solvent deuteron processes in two steps (Hvidt \& Nielsen, 1966). First, the site becomes transiently exposed to the solvent in a process with an equilibrium constant termed $K_{\text {open }}$. Second, the transiently exposed site exchanges with the solvent with an intrinsic rate constant, $k_{\text {int }}$, determined by the local sequence and the experimental conditions. After a rapid transient, the exchange process can be described by a pseudo-first order reaction with a rate constant, $k_{\mathrm{ex}}$, so that the probability, $P_{\mathrm{ex}}$, of a backbone hydrogen having exchanged with a hydrogen atom from the solvent becomes:

$$
P_{\text {ex }}(t)=1-e^{-k_{\text {ex }} t}
$$

where $t$ is the time elapsed since the exposure to $\mathrm{D}_{2} \mathrm{O}$.

Under relatively general assumptions and experimental conditions (leading to the so-called EX2 regime (Hvidt \& Nielsen, 1966), the observed exchange rate, $k_{\text {ex }}$, is simply the product of the equilibrium constant for transient opening, $K_{\text {open }}$, and 
the intrinsic rate, $k_{\text {int }}$. This relationship is typically formulated through the so-called protection factor, $P_{f}=K_{\text {open }}^{-1}$ :

$$
k_{\mathrm{ex}}=\frac{k_{\mathrm{int}}}{P_{f}}
$$

The structural dependencies of the exchange rate are thus captured through differences in protection factors. Here we describe these using a model originally presented by Vendruscolo and colleagues (Vendruscolo et al., 2003) in which the level of protection is predicted from examining the number of contacts and hydrogen bonds formed at an exchanging site:

$$
\log \left(P_{f}\right)=\beta_{h} N_{h}+\beta_{c} N_{c}
$$

where $N_{h}$ is the number of hydrogen bonds to the backbone nitrogen/hydrogen, and $N_{c}$ is the number of heavy atoms within a distance of $6.5 \AA$ of the backbone nitrogen. For atomistic models, the coefficients $\beta_{h}=2.0$ and $\beta_{c}=0.35$ were refined from an variety of molecular dynamics (MD) simulations and data from hydrogen exchange (HX) experiments (Best \& Vendruscolo, 2006). In the following examples and discussions, we estimate the presence of hydrogen bonds using the DSSP algorithm (Kabsch \& Sander, 1983) with a default energy threshold of $-0.5 \mathrm{kcal} / \mathrm{mol}$.

We show examples of protection factors predicted as described above on their respective atomic structures (Figure 2). As expected, we observe how well folded domains are more protected than flexible, solvent exposed regions. Furthermore, we note that transmembrane regions of membrane proteins are considered to be very well protected from the solvent; again in line with intuition. We note that in case of calculations for membrane proteins we embed the protein in a lipid bilayer or other membrane mimetic, and take contacts to these into account when calculating $P_{f}$.

The intrinsic exchange rate, $k_{\text {int }}$, represents the exchange rate for a fully unfolded peptide chain. It is readily estimated from the amino acid residue sequence (and IUCr macros version 2.1.10: 2016/01/28 
the experimental conditions) using e.g. the Sphere server (Zhang, 1995; Fox Chase Cancer Center, 2019), which bases its estimations on values found in the literature (Bai et al., 1993; Connelly et al., 1993) or the data published by the Englander Lab (Englander, 2019; Nguyen et al., 2018), which cites another source for the highpH exchange rates (Mori et al., 1997). The software presented in this paper uses the values from the latter two sources, which are refined from HX experiments on various reference peptides.

As an example, estimated intrinsic exchange rates for the sequence of lysozyme range from $0.03 \mathrm{~s}^{-1}$ to $46.60 \mathrm{~s}^{-1}$ across the 129 amino acid residues for a measured $p H$ of 7 at a temperature of $293.15 \mathrm{~K}$.

Apart from the backbone hydrogens, we assume that the following hydrogen atoms will exchange with the solvent at a timescale much faster than the ones observable by neutron scattering experiments due to their low $p K_{a}$ values (Lide, 2005):

- The - NHs in the N-terminal(s)

- The -OHs in SER, THR, and TYR

- The - SHs in CYS

- The side chain - NHs in ASN, GLN, ARG, LYS, HIS, and TRP

In other words, these chemical groups are assumed to instantly exchange hydrogen atoms with the solvent. We refer to these atoms as rapidly-exchanging throughout this paper. The set of rapidly-exchanging hydrogens and backbone hydrogens are sometimes referred to as the "labile" hydrogens in the literature. We note that in some cases exchange of the single - NH group in the indole group of TRP residues may be rather slow (Wedin et al., 1982), but since TRP residues are rather rare and there is no quantitative model for its exchange, we consider it as rapidly exchanging. The remaining hydrogens are considered non-exchangeable (Figure 1).

As an example of the result of these experimentally-supported assumptions, out 
of the 959 hydrogen atoms generated by PDB2PQR (Dolinsky et al., 2004; Dolinsky et al., 2007) for the PDB ID 2LYZ (Diamond, 1974) structure of lysozyme at a measured $p H$ of 7.0, we find 148 rapidly-exchangeable, 126 backbone hydrogens (for a total of 274 labile hydrogens), and the remaining 685 non-exchangeable.

\section{Solution small-angle neutron scattering}

\subsection{Estimating scattering from a protein in solution}

For the calculations of the scattering profiles (i.e. the excess scattered intensity, $I(q)$, as a function of the scattering momentum transfer, $q$ ) presented here, we have used a simple fast Debye sum (Hansen, 1990) based on commonly used scattering lengths (NIST, 2019) and volumes (Fraser et al., 1978) (Table 1). We stress that these values are central in our calculations. We assume a partial specific volume of solvent $\mathrm{D}_{2} \mathrm{O}$ of $30.0 \AA^{3}$. Details of these calculations can be found in Appendix A.

We note that more advanced and involved methods for estimating scattering profiles from the atomic coordinates exist; e.g. expansion of scattering amplitudes on spherical harmonics as employed by the software CRYSON (Svergun et al., 1998) or Debye sums as employed by the software FoXS (Schneidman-Duhovny et al., 2010) (for xray scattering). However, the simple, fast Debye method we use appears to be sufficient for our purposes, ranges in scattering momentum transfer, and structures.

\subsection{Comparison of time scales: Lysozyme}

As a first example, let us consider the application of the presented scheme in a Gedanken experiment: a generic solution SANS experiment on lysozyme at room temperature, $293.15 \mathrm{~K}$, in a $\mathrm{D}_{2} \mathrm{O}$-based buffer with a measured $p H$ of 7.0. In Figure 3, a calculation of the scattering profile at selected times is shown, along with the amount of backbone hydrogens expected to have exchanged with the solvent.

IUCr macros version 2.1.10: 2016/01/28 
We observe that in order for the imagined lysozyme to obtain fully or even $90 \%$ deuterated backbone hydrogens, the relevant time scales are orders of magnitude beyond those relevant in an experimental experiment. Indeed, the model predicts that the $90 \%$ backbone hydrogen deuteration commonly assumed, would not be reached until between $10^{9} \mathrm{~s}$ and $10^{10} \mathrm{~s}$ or a timespan of more than 30 years under the defined conditions. As expected, the excess scattering cross-section for vanishing values of $q$, usually dubbed "the forward scattering", decreases as the protein deuterates; as the exchange process brings the scattering length density of the protein closer to that of $\mathrm{D}_{2} \mathrm{O}$. In total, the forward scattering drops to $62 \%$ of the initial value; see Figure 3 (top). It is particularly noteworthy that within typical experiment times of 1 hour and 1 day, the forward scattering intensity drops to, respectively, $98 \%$ and $94 \%$ of the initial value.

In particular, we note that of the 274 labile sites, only $54 \%$ are rapidly-exchanged, and after more than a day $\left(10^{5} \mathrm{~s}\right)$ of exposure to $\mathrm{D}_{2} \mathrm{O}$ the model by Vendruscolo et al. predicts that only a total of $61 \%$ of the 274 labile hydrogen atoms will have exchanged. This value should be considered in relation to the usually applied $90 \%$ discussed earlier. We note also many residues in lysozyme have experimentally-determined values of $k_{\mathrm{ex}}<10^{-7} \mathrm{~s}^{-1}$ at $p H 7.0$ (Pedersen et al., 1993). This is fully consistent with the very long exchange times obtained from the model calculations.

\subsection{Comparison to experimental data: $B S A$}

The data presented in Figure 4 were collected at the SANS instrument D22 (D22, 2019), Institut Laue-Langevin, in Grenoble, France. Four samples of bovine serum albumin (BSA) were prepared from the same $\mathrm{H}_{2} \mathrm{O}$-based stock solution at either $p H$ 7.3 or $p H 8.8$ measured by $p H$-meter and changed into two analogous $\mathrm{D}_{2} \mathrm{O}$-based buffers using a NAP-5 desalting column (GE healthcare) either 9 or 36 hours before 
irradiation. The experiment, including sample storage, was conducted at approximately $6{ }^{\circ} \mathrm{C}$. For further details on the sample preparation and the experimental set up, consult the appendix.

We used PROPKA (Olsson et al., 2011) and chain A of PDB ID 4F5S (Bujacz, 2012) to estimate 4564 hydrogens in the BSA monomer, at $p H 7.3$ ( $p D$ of 7.7 ) and 4560 at $p H 8.8$ ( $p D$ of 9.2). According to the discussed model for H/D-exchange, the structure contains 499 hydrogen sites which will exchange rapidly (at $p H 7.3$ and an additional 4 at $p H$ 8.8) with the $\mathrm{D}_{2} \mathrm{O}$-based solvent and 554 slowly exchanging backbone hydrogens, the exchange rates of which we estimate using the presented software.

From Figure 4, we conclude that quantitively we observe the expected behaviour. We see the forward scattering, $I(q \rightarrow 0)$, decreasing as a function of how long time the proteins have been exposed to $\mathrm{D}_{2} \mathrm{O}$ (Figure 4, insert). Similarly, we note that the high- $p H$ samples appear to have considerably lower forward scattering than the low- $p H$ samples, in line with the expectation of more rapid exchange in these samples (Table 2). We note also that the effect of varying the time is greater at $p H 8.8$ where exchange is faster than at $p H 7.3$.

The presented fits are calculated as described. However, to fit the data we had to fit a scaling parameter which refined to $1.05-1.14$ along with a small constant background for the four presented samples. While it is obviously unsatisfactory to have to fit these adjustment parameters, we consider the refined values to be well within the margin-of-error of the absolute calibration of our data.

\subsection{Comparison of homogenouos and site-specific deuteration: SERCA}

Let us consider the membrane protein sarco-/endoplasmatic reticulum $\mathrm{Ca}^{2+}$ ATPase (SERCA) in an equilibrium between the E1 state, from PDB ID 5XA7 (Norimatsu et al., 2017), and the E2 state, from PDB ID 5XAB (Norimatsu et al., 2017), described 
by an ensemble of structures in a carrier system, which is assumed to be 'invisible' in $\mathrm{D}_{2} \mathrm{O}$ as previously discussed (Midtgaard et al., 2018; Maric et al., 2014; Josts et al., 2018).

We generated the ensemble of E1 and E2 states of SERCA using two independent one-microsecond MD simulations with CHARMM36m force field (Huang et al., 2016) initiating from the aforementioned crystal structures inserted into a 1-palmitoyl2-oleoylphosphatidylcholine (POPC) membrane bilayer. The simulations used the TIP3P water model and a periodic box of $13 \mathrm{~nm}$ by $13 \mathrm{~nm}$ by $16 \mathrm{~nm}$ and were performed using Gromacs2016.3 (Abraham et al., 2015). In total, we generate an ensemble of 40 structures describing the intermediate states between the aforementioned crystal structures. As previously described we average the calculated protection factors over these structures (Best \& Vendruscolo, 2006):

$$
\log \left(P_{f}\right)=\left\langle\beta_{h} N_{h}+\beta_{c} N_{c}\right\rangle
$$

and show calculated scattering profiles for the described structure under various conditions (Figure 5) at a measured $p H$ of 7.0 and a temperature of $293.15 \mathrm{~K}$.

We observe how the model predicts that the extra-cellular domains of the protein exchange faster than the transmembrane parts buried in the bilayer and how the effect of specifically exchanging specific sites mostly impacts the shape of the scattering profile for $q>0.1 \AA^{-1}$. In line with intuition, we observe that the difference between homogeneous deuteration and specific exchange matters most when the solvent-exposed domains of the proteins are mostly exchanged, while the transmembrane domains are still mostly protonated; i.e. the situation, wherein the protein appears mostly like a two-contrast molecule.

IUCr macros version 2.1.10: 2016/01/28 


\section{Software}

\subsection{Input and output}

As input, the software takes the atomic coordinates in PDB-format (Bernstein et al., 1977) of a protein alongside the coordinates of any other atoms relevant to the calculation e.g. a lipid bilayer for a membrane protein or bound ligands. We emphasize that unique model and chain identifiers specifying the individual models and molecules must be specified in the file as the calculation of $k_{\text {int }}$ depends on the chain structure of the protein. Several additional, optional arguments can be supplied for the calculation. A list of the most important optional arguments are listed in Table 3.

The intrinsic exchange rates, $k_{\text {int }}$, are calculated from the reference data published by the Englander Lab (Englander, 2019; Nguyen et al., 2018). As default, the software uses the reference values for poly-DL-Alanine; however, using their reference data from oligopeptides in the calculations is available as an alternative via a command line argument.

Whether hydrogens are present in the provided structure or not is irrelevant to the output. The software will remove any hydrogens from the structure and add them using the PDB2PQR software as well as the PROPKA algorithm.

The software outputs two files containing the provided structure. In one, values of $k_{\text {int }}$ are exported as the B-factor column and values of $\log \left(P_{f}\right)$ are exported as the occupancy column. The other file contains $\log \left(k_{\mathrm{ex}}\right)$ as the B-factors and $P_{\text {ex }}$ as the occupancies. In the second structure, hydrogen atoms will have been exchanged to deuterium atoms if $P_{\text {ex }}$ exceeds a preset threshold for the individual residues.

If several models are present in the given file (separated using the MODEL keyword), the protection factor calculation is done for each individual model and outputted. The protection factors are subsequently averaged as outlined in equation 1 , and each model is deuterated according to this average in an effort to simplify using the software on 
e.g. ensembles from nuclear magnetic resonance or MD trajectories.

\subsection{Code, dependencies, and availability}

Source code for the presented software is available for download or cloning from this Gitlab repository:

$$
\text { https://gitlab.com/mcpe/PSX }
$$

The software is published under version 3 of the GNU Publishing License, GPLv3, and is available 'as is'.

The software is written in Python and relies on the DSSP software (Kabsch \& Sander, 1983) for identifying hydrogen bonds and the PDB2PQR software for adding hydrogens to the structure based on the PROPKA algorithm. The reading and writing of the protein structures are handled using the PDB-tools (Hamelryck \& Manderick, 2003) in the BioPython package (Cock et al., 2009). As such, these modules must be available for the software to run correctly.

In passing, we mention that for the examples shown in this paper the running time of the software is a couple of seconds on an ordinary laptop. The software has been tested on Ubuntu and OSX.

\section{Perspectives and conclusions}

We have presented software to estimate the effects of solvent exposure using well established models and assessed this effect in the context of small-angle neutron scattering. Future investigations will attempt to estimate these effects in the context of other scattering techniques; most notably neutron reflectometry, as this technique would be susceptible to and affected by inaccurate estimates of scattering length (densities). For neutron reflectometry in particular, $\mathrm{H}_{2} \mathrm{O} / \mathrm{D}_{2} \mathrm{O}$-contrast variations are done in 
situ and is usually an integrated part of the experiment. Thus, the outlined effects are especially relevant in this context.

Given recent developments and experiences in biological small-angle neutron scattering methods, we believe more precises approaches to the problem of solvent exchange are needed in the neutron scattering community in order to utilize the experimental set ups and the acquired data fully.

The model that we use to estimate protection factors could be optimized, including improved values of $\beta_{c}$ and $\beta_{h}$. Refining optimal values for these parameters is an ongoing endeavour (Mohammadiarani et al., 2018) and future versions of the software might utilize different values, or indeed functional forms, than the one used here.

Finally, we observe that the default assumption of $90 \%$ deuteration of proteins in SANS experiments appears to be a very high estimate for generic, modern BioSANS samples according to the considered model and typical temperatures and values of $p H$ in experiments. Values between $50 \%$ and $70 \%$ seem more appropriate given our examples; however, ideally, these calculations should be repeated for each sample in an experiment. We hope the presented software will aid users in estimating this parameter.

\section{Appendix A Calculating scattering profiles}

We use the Fast Debye sum approach to calculating scattering profiles begins by establishing a scattering-weighted pair distance distribution over $N$ scatterers (Hansen, 
1990):

$$
p(r)=4 \pi \sum_{j}^{N} \sum_{k}^{N} \delta\left(r-r_{j k}\right) b_{j} b_{k}
$$

where $b_{j}$ and $b_{k}$ are the (excess) scattering lengths of the $j$ 'th and $k^{\prime}$ th scatterer, and $r_{j k}$ is the distance between them. In practice, this expression is usually binned into the data structure of a histogram. From this construction, we can calculate the scattering profile from the scatterers with a Fourier transformation:

$$
I(q)=4 \pi n \int_{0}^{\infty} d r p(r) \frac{\sin (q r)}{q r}
$$

where $n$ is the number density of the sample.

This method is readily extended for the structures emerging from the presented software.

If we (using e.g. the presented software) associate an exchange probability, $P_{\mathrm{ex}}$, to each backbone hydrogen (and set $P_{\mathrm{ex}}=0$ for all other scatterers), we get a concise expression by assigning two scattering lengths, $b^{\mathrm{H}}$ and $b^{\mathrm{D}}$, to each scatterer, where $b^{\mathrm{H}}$ is the scattering length in the case where the backbone hydrogen has not exchanged, and $b^{\mathrm{D}}$ is the scattering length elsewise. Note that $b^{\mathrm{H}}=b^{\mathrm{D}}$ for the scatterers that are not backbone hydrogens.

If we assume that the $P_{\mathrm{ex}} \mathrm{s}$ are statistically independent, we can construct a substitute for equation 2 :

$$
\begin{aligned}
p(r)= & 4 \pi \sum_{j}^{N} \sum_{k}^{N} \delta\left(r-r_{j k}\right) \cdot \\
& \left(b_{j}^{\mathrm{H}} b_{k}^{\mathrm{H}}\left(1-P_{\mathrm{ex}, j}\right)\left(1-P_{\mathrm{ex}, k}\right)+b_{j}^{\mathrm{H}} b_{k}^{\mathrm{D}}\left(1-P_{\mathrm{ex}, j}\right) P_{\mathrm{ex}, k}+\right. \\
& \left.b_{j}^{\mathrm{D}} b_{k}^{\mathrm{H}} P_{\mathrm{ex}, j}\left(1-P_{\mathrm{ex}, k}\right) \quad+b_{j}^{\mathrm{D}} b_{k}^{\mathrm{D}} P_{\mathrm{ex}, j} P_{\mathrm{ex}, k}\right)
\end{aligned}
$$

The Fourier transformation can now be carried out using equation 3.

\section{Appendix B}




\section{Preparation and handling of BSA samples}

\section{B.1. Sample preparation}

BSA (Sigma Aldrich) was dissolved in gel filtration buffer (20 mM Tris-HCl, $p H$ 7.5, $100 \mathrm{mM} \mathrm{NaCl}$ ) and applied to a Superdex 200 Increase (GE Healthcare) equilibrated in gel filtration buffer. Fractions spanning the peak corresponding to monomeric BSA were pooled and kept on ice until exchange into $\mathrm{D}_{2} \mathrm{O}$. The sample was split in four and changed into $\mathrm{D}_{2} \mathrm{O}$ using a NAP-5 desalting column (GE healthcare) equilibrated in $20 \mathrm{mM}$ Tris-DCl, $100 \mathrm{mM} \mathrm{NaCl}$ at either $p H 7.3$ or 8.8 (both $100 \% \mathrm{D} 2 \mathrm{O}$ ) and either 9 or 36 hours before irradiation.

\section{B.2. Experimental conditions}

The samples were irradiated by neutrons with a nominal wavelength of $6.0 \AA$ from a distribution with a width of $0.6 \AA$ in two experimental settings: one with a collimation length of $2.8 \mathrm{~m}$ and a sample-to-detector distance of $1.5 \mathrm{~m}$, and one with a collimation length of $8.0 \mathrm{~m}$ with a sample-to-detector distance of $8.0 \mathrm{~m}$ covering a range in scattering momentum transfer from $0.0084 \AA^{-1}$ to $0.57 \AA^{-1} .1 \mathrm{~mm}$ rectangular Hellma Suprasil quartz cuvettes (Hellma Analytics) were usied as sample containers during the experiment.

Furthermore, absorption at $280 \mathrm{~nm}$ was measured on the irradiated samples using a Nanodrop 1000 spectrophotometer (Thermo Fisher Scientific), from which we estimate the protein concentration of the irradiated fraction. The extinction coefficient at 280 $\mathrm{nm}$ for a BSA monomer was calculated to be $42925 \mathrm{M}^{-1} \mathrm{~cm}^{-1}$.

Instrumental effect were accounted for as described in the literature (Pedersen 
et al., 1990) using the values produced by GRASP (Dewhurst, 2019), which was also used to reduce the data and bring them to absolute units using the direct beam chopped by an ultra-fast chopper as reference. The presented models were calculated and instrumental effects were taken into account using WillItFit (Pedersen et al., 2013).

We thank the staff at Institut Laue-Langevin, Grenoble, France for the beam time and support resulting in the presented data, and thank Nicolai Tidemand Johansen for aiding in the preparation and irradiation of the BSA sample. We thank Raul Araya-Secchi for discussions on general implementations of the selected algorithms. This research was supported by the Lundbeck Foundation BRAINSTRUC initiative in structural biology, and the Synergy project funded by the Novo Nordisk foundation. NVidia's Academic Grant Program is acknowledged for granting the Titan Xp GPU on which many of the presented calculations were performed.

\section{References}

Abraham, M. J., Murtola, T., Schulz, R., Páll, S., Smith, J. C., Hess, B. \& Lindah, E. (2015). SoftwareX, 1-2, 19-25.

Bai, Y., Milne, J., Mayne, L. \& Englander, W. (1993). Proteins, 17(1), 75-86.

Bernstein, F. C., Koetzle, T. F., Williams, G. J., Meyer, E. F., Brice, M. D., Rodgers, J. R., Kennard, O., Shimanouchi, T. \& Tasumi, M. (1977). Eur. J. Biochem. 80(2), 319-324.

Best, R. B. \& Vendruscolo, M. (2006). Structure, 14(1), 97-106.

Bugge, K., Papaleo, E., Haxholm, G. W., Hopper, J. T. S., Robinson, C. V., Olsen, J. G., Lindorff-Larsen, K. \& Kragelund, B. B. (2016). Nat. Commun. 7(May), 11578.

Bujacz, A. (2012). Acta Crystallogr. Sect. D Biol. Crystallogr. 68(10), 1278-1289.

Cock, P. J., Antao, T., Chang, J. T., Chapman, B. A., Cox, C. J., Dalke, A., Friedberg, I., Hamelryck, T., Kauff, F., Wilczynski, B. \& De Hoon, M. J. (2009). Bioinformatics, 25(11), 1422-1423.

Connelly, G. P., Bai, Y., Jeng, M. F. \& Englander, S. W. (1993). Proteins, 17(1), 87-92.

D22, (2019). D22 - Large dynamic range small-angle diffractometer. https://www.ill.eu/users/instruments/instruments-list/d22/description/instrumentlayout/.

Dewhurst, C., (2019). GRASP - Graphical Reduction and Analysis SANS Program. http://www.ill.fr/lss/grasp/.

Diamond, R. (1974). J. Mol. Biol. 82(3), 371-391.

Dolinsky, T. J., Czodrowski, P., Li, H., Nielsen, J. E., Jensen, J. H., Klebe, G. \& Baker, N. A. (2007). Nucleic Acids Res. 35, 522-525.

Dolinsky, T. J., Nielsen, J. E., McCammon, J. A. \& Baker, N. A. (2004). Nucleic Acids Res. 32, $665-667$.

IUCr macros version 2.1.10: 2016/01/28 
Dutzler, R., Campbell, E. B. \& MacKinnon, R. (2003). Science, 108(2003), 108-112.

Englander, W., (2019). The Englander Lab Website, University of Pennsylvania. http://hx2.med.upenn.edu/.

Fox Chase Cancer Center, (2019). Sphere - A Server Program for Hydrogen Exchange Rate Estimation. http://landing.foxchase.org/research/labs/roder/sphere/sphere.html.

Fraser, R., MacRae, T. \& Suzuki, E. (1978). J. Appl. Crystallogr. 11, 693-694.

Granier, T., Gallois, B., Dautant, A., D'Estaintot, B. L. \& Précigoux, G. (1997). Acta Crystallogr. Sect. D Biol. Crystallogr. 53(5), 580-587.

Guinier, A. \& Fournet, G. (1955). Small-angle scattering of X-rays. John Wiley and Sons, New York.

Hamelryck, T. \& Manderick, B. (2003). Bioinformatics, 19(17), 2308-2310.

Hansen, S. (1990). J. Appl. Crystallogr. 23(4), 344-346.

Huang, J., Rauscher, S., Nawrocki, G., Ran, T., Feig, M., De Groot, B. L., Grubmüller, H. \& MacKerell, A. D. (2016). Nat. Methods, 14(1), 71-73.

Hvidt, A. \& Nielsen, S. O. (1966). In Advances in protein chemistry, vol. 21, pp. 287-386. Elsevier.

ISIS, (2019). Biomolecular Scattering Length Density Calculator. http://psldc.isis.rl.ac.uk/Psldc/.

Johansen, N. T., Pedersen, M. C., Porcar, L., Martel, A. \& Arleth, L. (2018). Acta Crystallogr. Sect. D Biol. Crystallogr. p. Accepted.

Jordan, A., Jacques, M., Merrick, C., Devos, J., Forsyth, V. T., Porcar, L. \& Martel, A. (2016). J. Appl. Crystallogr. 49(6), 2015-2020.

Josts, I., Nitsche, J., Maric, S., Mertens, H. D., Moulin, M., Haertlein, M., Prevost, S., Svergun, D. I., Busch, S., Forsyth, V. T. \& Tidow, H. (2018). Structure, 26, 1-8.

Kabsch, W. \& Sander, C. (1983). Biopolymers, 22(12), 2577-2637.

Kynde, S., Skar-Gislinge, N., Pedersen, M. C., Midtgaard, S. R., Simonsen, J. B., Schweins, R., Mortensen, K. \& Arleth, L. (2014). Acta Crystallogr. Sect. D Biol. Crystallogr. 70(2), $371-383$.

Lide, D. R. (ed.) (2005). CRC Handbook of Chemistry and Physics. CRC Press, Boca Raton, Florida.

Maric, S., Skar-Gislinge, N., Midtgaard, S., Thygesen, M. B., Schiller, J., Frielinghaus, H., Moulin, M., Haertlein, M., Forsyth, V. T., Pomorski, T. G. \& Arleth, L. (2014). Acta Crystallogr. Sect. D Biol. Crystallogr. 70(2), 317-328.

Midtgaard, S. R., Darwish, T. A., Pedersen, M. C., Huda, P., Larsen, A. H., Jensen, G. V., Kynde, S. A. R., Skar-Gislinge, N., Nielsen, A. J. Z., Olesen, C., Blaise, M., Dorosz, J. J., Thorsen, T. S., Venskutonyté, R., Krintel, C., Møller, J. V., Frielinghaus, H., Gilbert, E. P., Martel, A., Kastrup, J. S., Jensen, P. E., Nissen, P. \& Arleth, L. (2018). FEBS J. 285(2), 357-371.

Mohammadiarani, H., Shaw, V. S., Neubig, R. R. \& Vashisth, H. (2018). J. Phys. Chem. B, 122(1), 9314-9323.

Mori, S., van Zijl, P. C. \& Shortle, D. (1997). Proteins, 28(3), 325-32.

Nguyen, D., Mayne, L., Phillips, M. C. \& Walter Englander, S. (2018). J. Am. Soc. Mass Spectrom. 29(9), 1936-1939.

NIST, (2019). Neutron scattering lengths and cross sections. https://www.ncnr.nist.gov/resources/n-lengths/.

Norimatsu, Y., Hasegawa, K., Shimizu, N. \& Toyoshima, C. (2017). Nature, 545(7653), 193198.

Olsson, M. H. M., Sondergaard, C. R., Rostkowski, M. \& Jensen, J. H. (2011). J. Chem. Theory Comput. 7, 525-537.

Pedersen, J., Posselt, D. \& Mortensen, K. (1990). J. Appl. Crystallogr. 23(4), 321-333.

Pedersen, M. C., Arleth, L. \& Mortensen, K. (2013). J. Appl. Crystallogr. 46(6), 1894-1898. 
Pedersen, T. G., Thomsen, N. K., Andersen, K. V., Madsen, J. C. \& Poulsen, F. M. (1993). J. Mol. Biol. 230(2), 651-660.

Schneidman-Duhovny, D., Hammel, M. \& Sali, A. (2010). Nucleic Acids Res. 38(Web Server issue), $540-544$.

Svergun, D. I., Richard, S., Koch, M. H., Sayers, Z., Kuprin, S. \& Zaccai, G. (1998). Proc. Natl. Acad. Sci. U. S. A. 95(5), 2267-2272.

The SASView Project, (2019). SASView for Small Angle Scattering Analysis. https://www.sasview.org.

Vendruscolo, M., Paci, E., Dobson, C. M. \& Karplus, M. (2003). J. Am. Chem. Soc. 125(51), $15686-15687$.

Wedin, R. E., Delepierre, M., Dobson, C. M. \& Poulsen, F. M. (1982). Biochemistry, 21(5), $1098-1103$.

Williams, T., Kelley, C. et al., (2019). Gnuplot 5.2: an interactive plotting program. http://www.gnuplot.info/.

Zhang, Y.-Z. (1995). Protein and peptide structure and interactions studied by hydrogen exchange and NMR. Ph.D. thesis, University of Pennsylvania.

Table 1. The (coherent) scattering lengths and volumes used in the calculation of scattering profiles for proteins presented in this paper. The values are from NIST (NIST, 2019) and the

literature (Fraser et al., 1978), respectively.

\begin{tabular}{lll} 
Atom & Scattering length, fm & Assigned volume, $\AA^{3}$ \\
\hline H & -3.7406 & 5.15 \\
D & 6.671 & 5.15 \\
C & 6.646 & 16.44 \\
N & 9.36 & 2.49 \\
O & 5.803 & 9.13 \\
S & 2.847 & 19.86
\end{tabular}

Table 2. Concentration, $c$, radius of gyration, $R_{g}$, and normalized forward scattering, $I(q \rightarrow 0) / c$ for the data presented in Figure 4. The Guinier fits (Guinier E Fournet, 1955) were done using Gnuplot (Williams et al., 2019).

\begin{tabular}{|c|c|c|c|}
\hline Sample & $c, \mathrm{mg} / \mathrm{ml}$ & $R_{g}, \stackrel{\circ}{A}$ & $I(q \rightarrow 0) / c, \mathrm{~cm}^{2} / \mathrm{mg}$ \\
\hline$\square$ & 1.18 & $25.8 \pm 0.31$ & $0.1280 \pm 0.00072$ \\
\hline $\mathbf{\square}$ & 1.24 & $26.0 \pm 0.26$ & $0.1336 \pm 0.00063$ \\
\hline [ & 1.30 & $25.7 \pm 0.25$ & $0.1460 \pm 0.00067$ \\
\hline 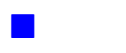 & 1.32 & $26.2 \pm 0.26$ & $0.1476 \pm 0.00071$ \\
\hline
\end{tabular}


Table 3. Selected optional command line arguments.

\begin{tabular}{|c|c|c|}
\hline Argument & Description & Default value \\
\hline$-\mathrm{bc}$ & $\begin{array}{l}\text { The coefficient } \beta_{c} \text { used to } \\
\text { calculate protection factors }\end{array}$ & 0.35 \\
\hline$-b h$ & $\begin{array}{l}\text { The coefficient } \beta_{h} \text { used to } \\
\text { calculate protection factors }\end{array}$ & 2.0 \\
\hline$-d c$ & $\begin{array}{l}\text { Distance threshold used in the } \\
\text { calculation of the contact } \\
\text { number, } N_{c} \text {. in } \AA\end{array}$ & 6.5 \\
\hline$-e h$ & $\begin{array}{l}\text { Energy threshold for hydrogen } \\
\text { bond energy in DSSP algorithm in } \\
\text { kcal/mol }\end{array}$ & -0.5 \\
\hline$-\mathrm{ph}$ & $\begin{array}{l}p H \text { used to estimate exchange } \\
\text { rates as measured by } p H \text {-meter }\end{array}$ & 7.0 \\
\hline$-p c$ & $\begin{array}{l}\text { Probability threshold above } \\
\text { which hydrogens will be } \\
\text { exchanged in the output structure }\end{array}$ & 0.5 \\
\hline$-t i$ & $\begin{array}{l}\text { Time elapsed since the protein } \\
\text { was exposed to } \mathrm{D}_{2} \mathrm{O} \text { in seconds }\end{array}$ & 0.0 \\
\hline -te & $\begin{array}{l}\text { Temperature used to intrinsic } \\
\text { estimate exchange rates in K }\end{array}$ & 293.15 \\
\hline
\end{tabular}

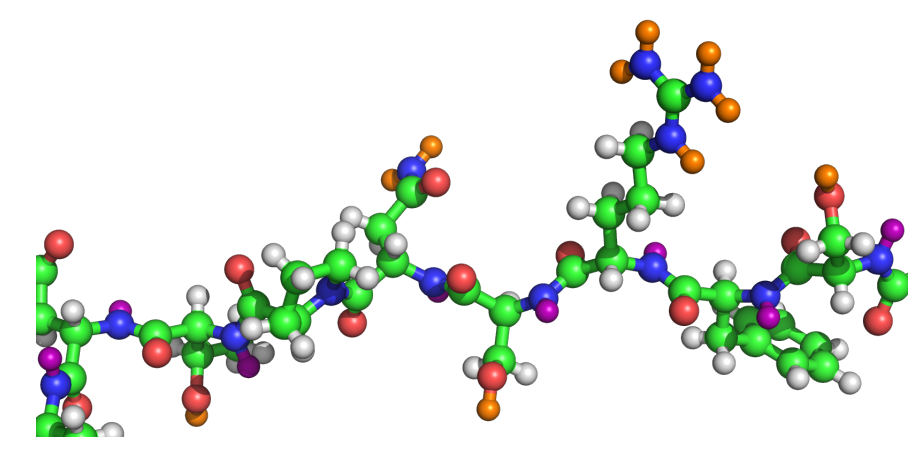

Fig. 1. A generic protein structure with backbone amide hydrogens highlighted in purple, un-exchangeable hydrogens in white, and the rapidly-exchanging hydrogens in orange. Carbon-, nitrogen-, and oxygen-atoms are shown in green, blue, and red, respectively. 


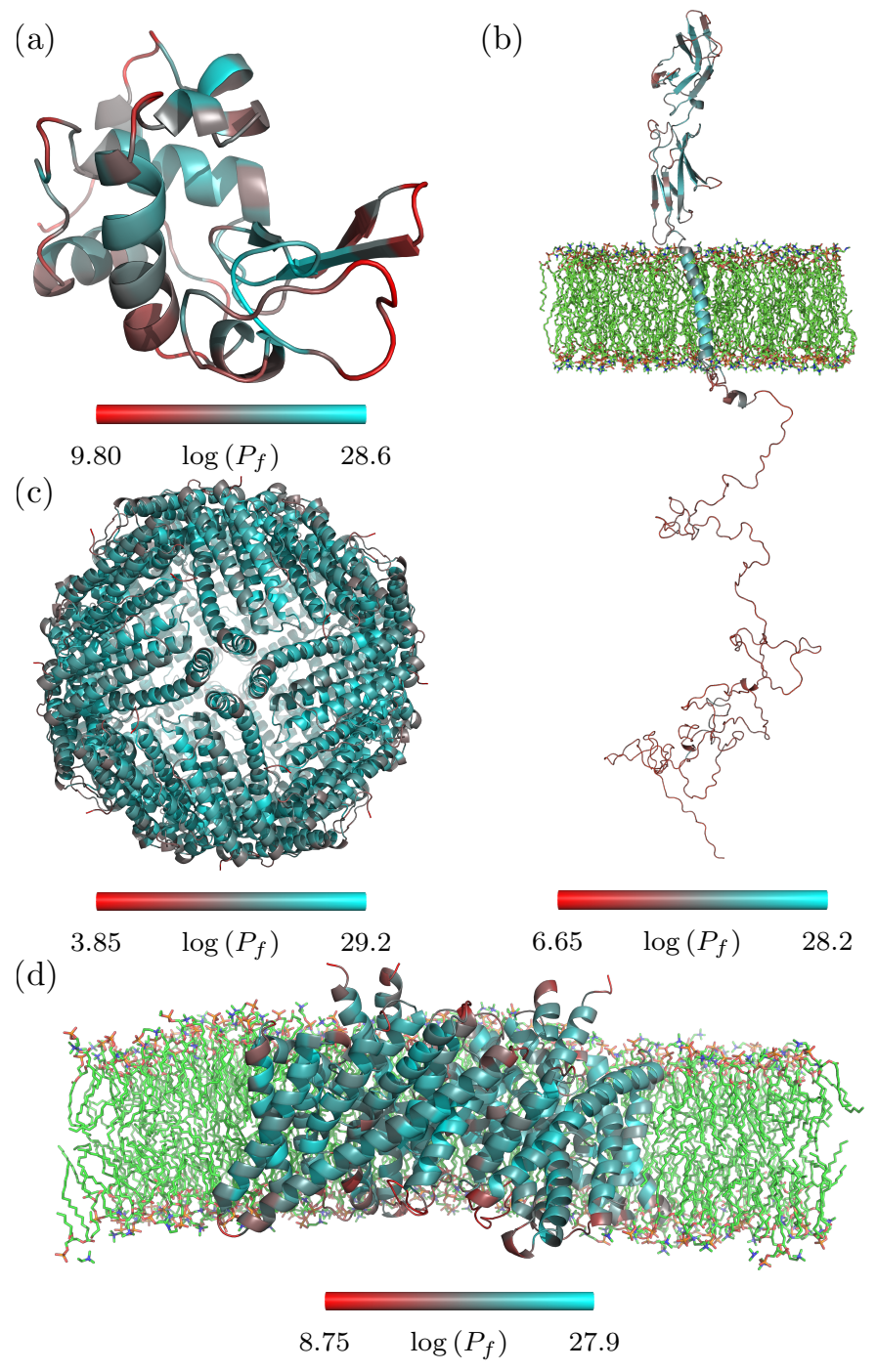

Fig. 2. Examples of protection factors calculated from protein structures. (a): Lysozyme, from PDB ID 2LYZ. (b): Prolactin receptor in a bilayer of POPC phospholipids (half of which are not shown), from Bugge et al. (Bugge et al., 2016). (c): Apoferritin (24-mer), from PDB ID 1IES (Granier et al., 1997). (d): $\mathrm{H}^{+} / \mathrm{Cl}^{-}$ Exchange Transporter in a bilayer of DPPC phospholipids (half of which are not shown), from PDB ID 1OTU (Dutzler et al., 2003). 

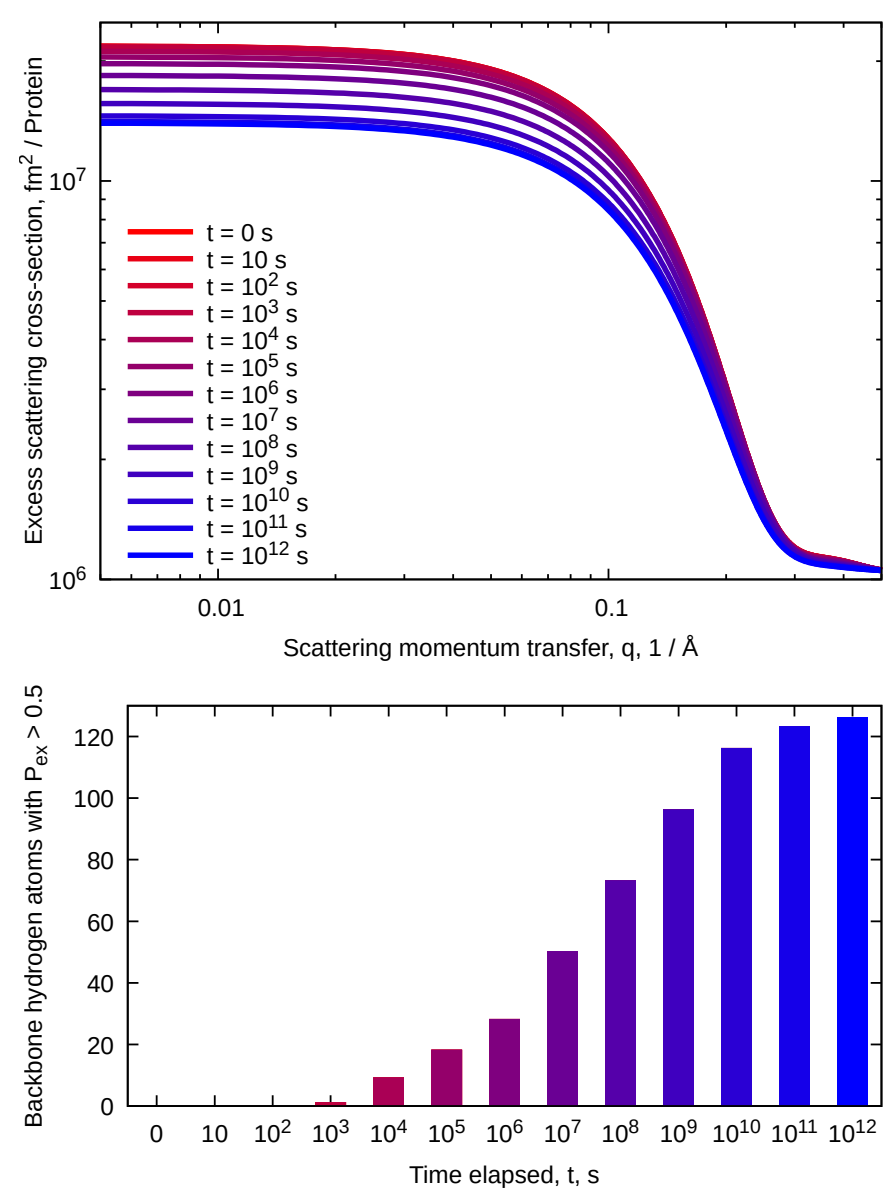

Fig. 3. Time evolution of the structure PDB ID 2LYZ, lysozyme, shown in Figure 2(a), according to the solvent exposure model discussed in this paper. Top: Scattering profiles calculated as explained in the text; a small background has been added the the profiles for a more realistic presentation. Bottom: The number of the 126 backbone hydrogens atoms with $P_{\mathrm{ex}}>0.5$ for selected time points. We remind the reader that $10^{5} \mathrm{~s}$ is approximately 1.15 days and that $10^{8} \mathrm{~s}$ is approximately 3.16 years. 


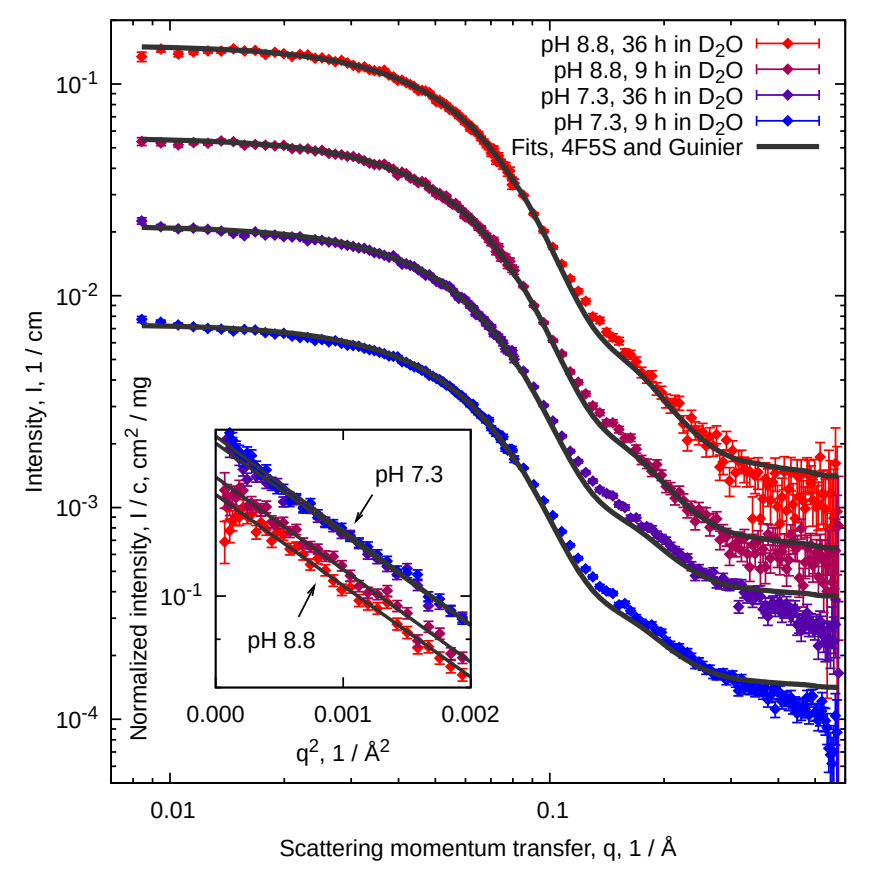

Fig. 4. SANS data from BSA. The top-most data and fit are correctly scaled, whereas the lower data and fits are scaled by factors of 3 relative to the one above. The insert shows Guinier fits. Selected parameters are listed in Table 2. 

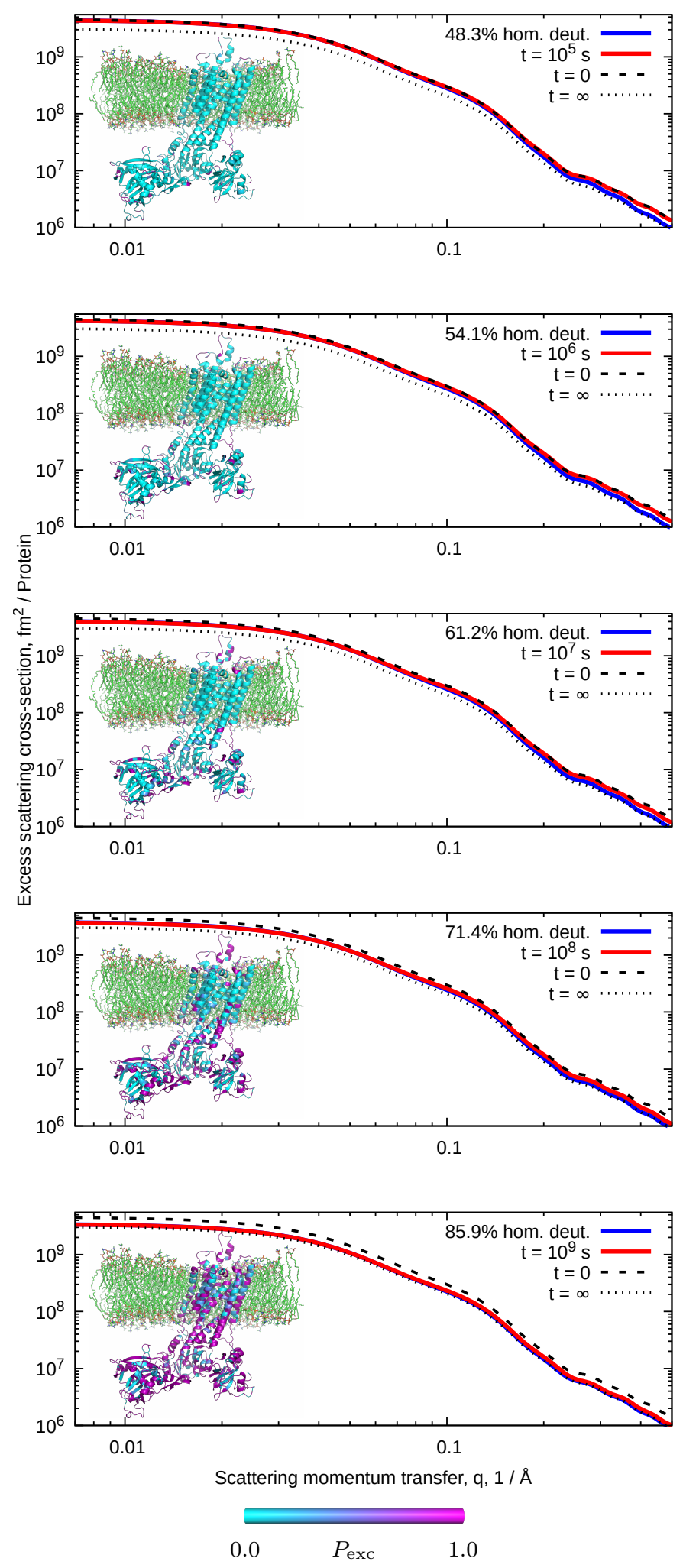

Fig. 5. Scattering profiles of SERCA. As inserts, we visualize $P_{\mathrm{ex}}$ on the E1 state of IUCSERGGA Aerian a 1bilayed/00,28POPC phospholipids (half of which are not shown). The phospholipids do not contribute to the scattering pattern in an effort to mimick the scattering from match-out deuterated carrier systems. For reference, we also plot the scattering profiles of the fully deuterated structure and the structure with only the rapidly-exchanging sites exchanged to deuterium. 


\section{Synopsis}

Using established models, we calculate the effect of exposing protonated protein samples to $\mathrm{D}_{2} \mathrm{O}$ and assess the impact in small-angle neutron scattering experiments. We introduce software to evaluate the effect of this from a given structure and give examples of applications. 